Pacific Journal of Mathematics

THE MINIMUM BOUNDARY FOR AN ANALYTIC 


\title{
THE MINIMUM BOUNDARY FOR AN ANALYTIC POLYHEDRON
}

\author{
Kenneth Hoffman AND Hugo Rossi
}

1. Introduction. If $K$ is a compact subset of a complex analytic manifold $M$, then for each $f$, holomorphic (analytic) in a neighborhood of $K$, the maximum modulus of $f$ over $K$ is attained on the topological boundary of $K$. If the complex dimension of $M$ is greater than 1 , it may happen that there are proper closed subsets of the topological boundary on which each holomorphic $f$ attains its maximum modulus. In case there are sufficiently many holomorhic functions on $M$ to separate the points of the manifold, a general result of Šilov [6] states that there is a uniquely determined smallest closed subset of $K$ which has this maximum modulus property. This set is known as the Silov boundary for the ring of functions holomorphic in a neighborhood of $K$.

The Silov theorem is valid for separating algebras of continuous complex-valued functions on a compact space, and has nothing to do with analyticity as such. Many years earlier, the pioneering work on maximum modulus sets for rings of analytic functions had been done by Bergman $[1 ; 2 ; 3]$. He considered principally domains in $C^{n}$ which were bounded by a finite number of analytic hypersurfaces, and for these he introduced a distinguished boundary surface. For a wide class of such domains, he showed that his distinguished boundary was a smallest maximum modulus set. References to more recent work on these problems may be found in the second author's paper [9].

In this paper we consider the case in which $M$ is a Stein manifold, e.g., a domain of holomorphy in $C^{n}$, and the compact set $K$ is an analytic polyhedron. This means that $K$ has the form

$$
K=\left\{m \in D ;\left|f_{j}\right| \leqq 1, j=1, \cdots, k\right\}
$$

where $f_{1}, \cdots, f_{k}$ are holomorphic functions on some open subset $D$ of the manifold $M$. We consider those subsets $S$ of $K$ which have the property that, for every $f$ holomorphic in a neighborhood of $K$, the maximum modulus of $f$ over $K$ is attained on the subset $S$. We prove that among all such subsets $S$ there is a smallest one, which we call the minimum boundary for the polyhedron. The closure of this minimum boundary is (of course) the Šilov boundary for the ring of functions holomorphic on $K$. While it is difficult to give an explicit description of this Śilov boundary, such a description can be given for the minimum boundary. It is obtained by deleting from $K$ all connected local analytic

Received May 16, 1962. 
varieties of positive dimension which are contained in $K$. In terms of the functions $f_{j}$ which define the polyhedron $K$, the description is as follows. Let $m_{0} \in K$, and let $j_{1}, \cdots, j_{r}$ be those indices $j$ for which $\left|f_{j}\left(m_{0}\right)\right|=1$. Then $m_{0}$ belongs to the minimum boundary if and only if $m_{0}$ is an isolated point of the set (variety)

$$
V=\left\{m \in D ; f_{j_{i}}(m)=f_{j_{i}}\left(m_{0}\right), i=1, \cdots, r\right\} .
$$

In [7], the first author identified the minimum boundary for the ring of functions on $K$ which are uniform limits of functions holomorphic in a neighborhood of $K$. The arguments there made essential use of a fundamental theorem of Bishop: if $A$ is a uniformly closed separating algebra of continuous complex-valued functions on a compact metric space $K(1 \in A)$, there is a smallest subset of $K$ on which each function in $A$ attains its maximum modulus. This minimum boundary for $A$ consists of the points of $K$ at which some function in $A$ "peaks", i.e., those points with the property that there is a function in $A$ which attains its maximum modulus at the point, and at no other point. The description of the minimum boundary for the ring of functions holomorphic on $K$ is exactly the one which was shown in [7] to define the minimum boundary for the uniform closure of the ring. In particular, it results that the minimum boundary for the polyhedron consists of those points of $K$ at which some holomorphic function peaks (over $K$ ), or, it consists of the peak points for functions which are uniformly approximable by holomorphic functions. We shall use methods from [7], and we shall make essential use of Bishop's general existence theorem. This theorem is not directly applicable to the ring of functions holomorphic in a neighborhood of $K$, since the ring is not generally closed under uniform convergence. However, by using a technique from the second author's paper [8], based upon the solution of the second Cousin problem for Stein manifolds, we are able to show that the ring of holomorphic functions has the same peak points as does its uniform closure.

2. Notation and basic definitions. A Stein manifold is a $d$-dimensional complex analytic manifold $M$ such that

(i) the global holomorphic (analytic) functions on $M$ separate the points of $M$;

(ii) for each point $m \in M$ there are global holomorphic functions $h_{1}, \cdots, h_{a}$ which serve as coordinates in some neighborhood of $m$;

(iii) $M$ is a countable union of compact sets;

(iv) if $K$ is any compact set in $M$, the set of points $m \in M$ such that $|h(m)| \leqq \sup _{K}|h|$ for every holomorphic function $h$ on $M$ is also compact. 
Let $M$ be a Stein manifold. An analytic polyhedron in $M$ is a subset $P$ of $M$ such that

(i) $P$ is compact

(ii) $P=\left\{m \in D ;\left|f_{j}(m)\right| \leqq 1, j=1, \cdots, k\right\}$, where $D$ is an open subset of $M$ and $f_{1}, \cdots, f_{k}$ are holomorphic functions on $D$.

If $P$ is an analytic polyhedron in $M$ we denote by $H(P)$ the set of all functions $f$ on $P$ such that $f$ is the restriction to $P$ of a function holomorphic in some neighborhood of $P$. We denote by $A(P)$ the class of functions on $P$ which can be approximated, uniformly on $P$, by functions in $H(P)$. Both $H(P)$ and $A(P)$ are algebras of continuous functions on $P$. Our task is to prove the existence of a smallest subset $S$ of $P$ such that, for every $h$ in $H(P)$, the maximum modulus of $h$ over $P$ is attained on the set $S$, and then to describe the set $S$ explicitly. For this we need to discuss briefly boundaries for algebras of continuous functions.

Let $X$ be a compact Hausdorff space, and let $A$ be a collection of continuous complex-valued functions on $X$. A boundary (of $X$ ) for $A$ is a subset $S$ of $X$ such that

$$
\max _{S}|f|=\max _{x}|f|, \quad f \in A
$$

that is, a subset $S$ of $X$ such that for each $f$ in $A$ the maximum modulus of $f$ over $X$ is attained at some point of $S$. If

(a) $A$ is a complex-linear algebra, using pointwise operations

(b) the constant functions are in $A$

(c) the functions in $A$ separate the points of $X$, then among all closed boundaries for $A$ there is a smallest one, i.e., the intersection of all closed boundaries for $A$ is a boundary for $A$. This smallest closed boundary for $A$ we call the Silov boundary for $A$, in honor of G. E. Šilov who first proved its existence [6]. If, in addition to (a), (b), and (c) we have

(d) $A$ is closed under uniform convergence

(e) $X$ is metrizable

then the intersection of all boundaries for $A$ is a boundary for $A$. This smallest of all boundaries we shall call the minimum boundary for $A$. Its existence was proved by Bishop [4], who also showed that it consists of those points $x \in X$ which are peak points for $A$. We call $x$ a peak point for $A$ if there exists an $f$ in $A$ such that $|f(x)|>|f(y)|$ for all points $y$ in $X$ which are different from $x$. Evidently, the Šlov boundary for $A$ is the closure of the minimum boundary for $A$.

Both $H(P)$ and $A(P)$ are algebras of continuous functions on the compact space $P$ which satisfy conditions (a), (b), (c) above. Since $A(P)$ is the uniform closure of $H(P)$, these algebras have the same Silov boundary. Now $P$ is metrizable, as is easy to see from the countability 
condition imposed on a Stein manifold. Therefore, there exists a minimum boundary for the uniformly closed algebra $A(P)$. The general function algebra results cited above do not guarantee the existence of a minimum boundary for $H(P)$; thus, as we proceed now to prove the existence of such a boundary, we shall make heavy use of explicit properties of analytic polyhedra.

3. The minimum boundary. Now suppose we are given the analytic polyhedron

$$
P=\left\{m \in D ;\left|f_{j}(m)\right| \leqq 1, j=1, \cdots, k\right\}
$$

in the Stein manifold $M$. With each point $m_{0}$ in the polyhedron $P$ we associate an analytic variety $V_{m_{0}}$ in the ambient neighborhood $D$ by

$$
V_{m_{0}}=\left\{m \in D ; f_{j_{i}}(m)=f_{j_{i}}\left(m_{0}\right), i=1, \cdots, r\right\}
$$

where $j_{1}, \cdots, j_{r}$ are those indices $j$ such that $\left|f_{j}\left(m_{0}\right)\right|=1$.

Theorem 1. Let $m_{0} \in P$ and suppose $m_{0}$ is a local peak point for the algebra $A(P)$. Then $m_{0}$ is an isolated point of the variety $V_{m_{0}}(3.1)$.

Proof. This is proved in [7; Theorem 4.1]; here, we merely outline the proof. By the statement that $m_{0}$ is a local peak point for the algebra $A(P)$ we mean that there is a function $h \in A(P)$ and a neighborhood $N$ of $m_{0}$ such that $f\left(m_{0}\right)=1$ and $|f(m)|<1$ for all other points in $N \cap P$. Given such an $f$ and $N$, we may assume that those functions $f_{j}$ (occurring in the definition of $P$ ) which are of modulus less than 1 at $m_{0}$ are of modulus less than 1 on the open set $N$. Then $N \cap V_{m_{0}}=$ $V$ is an analytic variety in $N$ and this variety is contained in the polyhedron $P$. Since $f$ is a uniform limit on $P$ of functions holomorphic in a neighborhood of $P, f$ is 'analytic' on the variety $V$. Also, $f$ has a local maximum over $V$ at the point $m_{0}$. The maximum modulus principle for analytic varieties then states that $m_{0}$ is an isolated point of $V$.

THEOREM 2. Let $m_{0} \in P$ and suppose that $m_{0}$ is an isolated point of the variety $V_{m_{0}}(3.1)$. Then $m_{0}$ is a peak point for the algebra $H(P)$.

Proof. Let $g_{i}=\frac{1}{2}\left(1+\overline{f_{j_{i}}\left(m_{0}\right)} f_{j_{i}}\right), i=1, \cdots, r$. Then the function $g_{i}$ is bounded by 1 on $P$, and has the value 1 at any point of $P$ where it is of modulus 1 . Now let $h=g_{1} \cdots g_{r}$. Then $h$ is bounded by 1 on $P$, has the value 1 at any point of $P$ where it is of modulus 1 , and, furthermore, the set of points in $P$ where $h$ has the value 1 is precisely the intersection of $P$ with the analytic variety $V_{m_{0}}$. Thus, $m_{0}$ is an 
isolated point of the set on which $h=1$. We should also remark that $h$ is holomorphic in the open set $D$ which occurs in the definition of $P$.

Since $M$ is a Stein manifold, we can find functions $h_{1}, \cdots h_{n}$, holomorphic on all of $M$, such that the map $m \rightarrow\left(h_{1}(m), \cdots, h_{n}(m)\right)$ is biholomorphic on $P$, and the image of $P$ under this map is a polynomial convex subset of $C^{n}$. See [5]. Now we consider the map

$$
\phi(m)=\left(h_{1}(m), \cdots, h_{n}(m), h(m)\right)
$$

from $D$ into $C^{n+1}$. This map is biholomorphic, and the set $K=\phi(P)$ is polynomial convex. (polynomial convexity of $K$ means that if $z$ is a point of $C^{n+1}$ which is not in $K$, there exists a polynomial $p$ in $(n+1)$ variables such that $|p(z)|>\sup _{K}|p|$.) Let $z^{0}=\phi\left(m_{0}\right)$. The coordinate function $z_{n+1}$ is bounded by 1 on $K$, is equal to one at any point of $K$ where it is of modulus 1 , and $z^{0}$ is an isolated point of $K \cap\left\{z_{n+1}=1\right\}$.

Choose a neighborhood $U$ of the point $z^{0}$ such that for any point $z=\left(z_{1}, \cdots, z_{n+1}\right)$ in $U \cap K$ which is different from $z^{0}$ we have $\left|z_{n+1}\right|<1$. By [8; Theorem 2.4] there exists a function $g$, holomorphic in a neighborhood $W$ of $K$, such that $g$ never vanishes on $W-U$ and $g /\left(1-z_{n+1}\right)$ is holomorphic and without zeros on $W \cap U$.

On the neighborhood $W \cap U$, the function $g$ has the form $g=$ $\left(z_{n+1}-1\right) k$, where $k$ is holomorhpic and never vanishes on $W \cap U$. In particular $k\left(z^{0}\right) \neq 0$. Thus, by shrinking $U$, we may assume that $k$ has a single-valued logarithm; say $k=e^{l}$ where $l$ is holomorphic on $W \cap U$. Since $K$ is polynomial convex, it is an intersection of polynomial convex open sets. Therefore, it may be assumed that $W$ is polynomial convex, and hence is itself a Stein manifold. Similarly, a small contraction of $W$ will assure that the part of the intersection of $W$ with the hyperplane $\left\{z_{n+1}=1\right\}$ which lies in $U$ is a closed analytic variety in $W$. Since $W$ is a Stein manifold, there is a holomorphic function $p$ on $W$ such that $p=l$ on that variety [5]. Now let $\widetilde{g}=g e^{-p}$. Then $\widetilde{g}$ is holomorphic on $W$ and has no zeros on $W-U$. Also $\widetilde{g}=\left(z_{n+1}-1\right) k e^{-p}$ on $W \cap U$. Now $k e^{-p}=k e^{-l}=1$ on that part of the hyperplane $\left\{z_{n+1}=1\right\}$ which lies in $W \cap U$. Thus, on $W \cap U, k e^{-p}=1+\left(z_{n+1}-1\right) \tilde{k}$. Finally we have a function $\widetilde{g}$, holomorphic on $W$, which has no zeros on $W-U$ and has the form

$$
\widetilde{g}=\left(z_{n+1}-1\right)+\left(z_{n+1}-1\right)^{2} \tilde{k}
$$

on $W \cap U(\widetilde{k}$ holomorphic on $W \cap U)$.

From $\widetilde{g}$ we shall now construct a function holomorphic in a neighborhood of $K$, which has the property that its maximum modulus over $K$ is attained at $z^{0}$ and at no other point of $K$. This function will be an analytic function of $\widetilde{g}$. Thus we shall examine the range of $\widetilde{g}$ on $K$. The crucial fact about the set $\widetilde{g}(K)$ is that it lies outside a simply 
connected domain in the plane which has an analytic boundary containing the origin. To see this, we argue as follows. Choose a neighborhood $N$ of $z^{0}$ such that the function $\tilde{k}$ is bounded on $W \cap N$. We shall then have

$$
\left|\widetilde{g}-\left(z_{n+1}-1\right)\right| \leqq c\left|z_{n+1}-1\right|^{2} \text { on } W \cap N
$$

where $c$ is some positive constant. The range of $\left(z_{n+1}-1\right)$ on $K$ lies in the left half-plane. For each point $w$ in the left halfplane, we consider the disc

$$
|\zeta-w| \leqq c|w|^{2} .
$$

It is easy to see that there is an analytic curve $\gamma$ through the origin such that for all points $w$ near the origin this disc lies to the left of $\gamma$. Because, a short computation shows that the envelope of the family of dises is an analytic curve through the origin. We may assume that $N$ is sufficiently small that for any point $z$ in $K \cap N$ the point $w=$ $z_{n+1}-1$ has this property. Therefore, the range of $\widetilde{g}$ on $N \cap K$ lies to the left of $\gamma$. On $K-N$ the function $\widetilde{g}$ has no zeros. Choose $\varepsilon>0$ such that $|\widetilde{g}|>\varepsilon$ on $K-N$. If $\varepsilon$ is sufficiently small, the circle $|w|=$ $\varepsilon$ will intersect $\gamma$ in precisely two points. Let $D$ be the domain bounded by $\gamma$ and $|w|=\varepsilon$. Let $\tau$ be the Rieman map of the complement of $D$ onto the unit disc, which carries the origin onto 1 . Since $\gamma$ is an analytic curve, $\tau$ will extend analytically across that part of $\gamma$ which is on the boundary of $D$. The composition $\tau \circ \widetilde{g}=F$ is then holomorphic on a neighborhood of $K$, is of modulus less than 1 on $K-\left\{z^{0}\right\}$, and $F\left(z^{0}\right)=1$.

Now we return to the polyhedron $P$ which we mapped holomorphically onto $K$ via the map $\phi$. If we let $f=F \circ \phi$, then $f$ is holomorphic in a neighborhood of $P$, and the maximum modulus of $f$ over $P$ is attained at $m_{0}$ and at no other point of $P$. Thus $m_{0}$ is a peak point for the algebra $H(P)$.

CoRollary. Let $M$ be a Stein manifold, and Let $P$ be an analytic polyhedron in $M$. Then there is a (unique) smallest subset $S$ of $P$ such that for every function $f$, holomorphic in a neighborhood of $P$, the maximum modulus of $f$ over $P$ is attained on the set $S$. A necessary and sufficient condition that a point $m_{0}$ in $P$ should belong to this minimum boundary $S$ is any one of the following.

(i) $m_{0}$ is a peak point for the algebra $H(P)$.

(ii) $m_{0}$ is a peak point for the algebra $A(P)$.

(iii) $m_{0}$ is a local peak point for the algebra $H(P)$.

(iv) $m_{0}$ is a local peak point for the algebra $A(P)$.

(v) There is no connected local analytic variety of positive dimension which passes through $m_{0}$ and is contained in $P$. 
(vi) $m_{0}$ is an isolated point of the variety $V_{m_{0}}$, defined by (3.1).

Proof. By Bishop's theorem [4], there is a minimum boundary for the algebra $A(P)$, and it consists of those points of $P$ which are peak point for $A(P)$. In Theorem 1 we showed that any local peak point for the algebra $A(P)$ satisfies (vi). Indeed, the proof showed that the point satisfies (v), which clearly implies (vi). Theorem 2 states that (vi) implies (i). From this it is clear that the six statements about $m_{0}$ are equivalent. Furthermore, it is evident that the minimum boundary for $A(P)$ is a boundary for $H(P)$; and, since each point of this boundary is a peak point for $H(P)$, this boundary is the smallest boundary for $H(P)$.

\section{BIBLIOGRAPHY}

1. S. Bergman, Über ausgezeichnete Randfläche in der Theorie der Funktionen von zwei komplexen Veränderlichen, Math. Ann., 104 (1931), 611.

2. — Über die Veranschanlichung der Kreiskörper und Bereiche mit ausgezeichneter Randfläche, Jahresber. d. d. Math. Ver., 42 (1933), 238.

3. — Über eine in gewissen Bereichen mit Maximumfäche gültige Integraldarstellung der Functionen zweier komplexer Variabler, Math. Z., 39 (1934), 76-94, 605-608.

4. E. Bishop, A minimal boundary for function algebras, Pacific J. Math., 9 (1959), 629.

5. Séminaires de H. Cartan, École Normale Supérieure, Paris, 1951-52.

6. Gelfand, I., Raikov, D., and Šilov, G., Commutative Normed Rings, A.M.S. Translations Series 2, vol. 5, 1957.

7. K. Hoffman, Minimal boundaries for analytic polyhedra, Rend. del Circ. Mat. di Palermo, ser. 2, $9(1960), 14^{\prime} 7$.

8. H. Rossi, The local maximum modulus principle, Ann. of Math., 71 (1960), 1.

9. - Holomorphically convex sets in several complex variables, Ann. of Math., 74 $(1961), 470$. 



\section{PACIFIC JOURNAL OF MATHEMATICS}

\section{EDITORS}

\author{
Ralph S. Phillips \\ Stanford University \\ Stanford, California \\ M. G. Arsove \\ University of Washington \\ Seattle 5 , Washington
}

\author{
A. L. Whiteman \\ University of Southern California \\ Los Angeles 7, California \\ Lowell J. Paige \\ University of California \\ Los Angeles 24, California
}
E. F. BECKENBACH
D. DERRY
ASSOCIATE EDITORS
T. M. CHERRY
M. OHTSUKA
H. L. ROYDEN
E. G. STRAUS
E. SPANIER
F. WOLF

\section{SUPPORTING INSTITUTIONS}

\author{
UNIVERSITY OF BRITISH COLUMBIA \\ CALIFORNIA INSTITUTE OF TECHNOLOGY \\ UNIVERSITY OF CALIFORNIA \\ MONTANA STATE UNIVERSITY \\ UNIVERSITY OF NEVADA \\ NEW MEXICO STATE UNIVERSITY \\ OREGON STATE UNIVERSITY \\ UNIVERSITY OF OREGON \\ OSAKA UNIVERSITY \\ UNIVERSITY OF SOUTHERN CALIFORNIA
}

\author{
STANFORD UNIVERSITY \\ UNIVERSITY OF TOKYO \\ UNIVERSITY OF UTAH \\ WASHINGTON STATE UNIVERSITY \\ UNIVERSITY OF WASHINGTON \\ AMERICAN MATHEMATICAL SOCIETY \\ CALIFORNIA RESEARCH CORPORATION \\ SPACE TECHNOLOGY LABORATORIES \\ NAVAL ORDNANCE TEST STATION
}

Mathematical papers intended for publication in the Pacific Journal of Mathematıcs should be typewritten (double spaced), and the author should keep a complete copy. Manuscripts may be sent to any one of the four editors. All other communications to the editors should be addressed to the managing editor, L. J. Paige at the University of California, Los Angeles 24, California.

50 reprints per author of each article are furnished free of charge; additional copies may be obtained at cost in multiples of 50 .

The Pacific Journal of Mathematics is published quarterly, in March, June, September, and December. Effective with Volume 13 the price per volume (4 numbers) is $\$ 18.00$; single issues, $\$ 5.00$. Special price for current issues to individual faculty members of supporting institutions and to individual members of the American Mathematical Society: $\$ 8.00$ per volume; single issues $\$ 2.50$. Back numbers are available.

Subscriptions, orders for back numbers, and changes of address should be sent to Pacific Journal of Mathematics, 103 Highland Boulevard, Berkeley 8, California.

Printed at Kokusai Bunken Insatsusha (International Academic Printing Co., Ltd.), No. 6, 2-chome, Fujimi-cho, Chiyoda-ku, Tokyo, Japan.

PUBLISHED BY PACIFIC JOURNAL OF MATHEMATICS, A NON-PROFIT CORPORATION

The Supporting Institutions listed above contribute to the cost of publication of this Journal, but they are not owners or publishers and have no responsibility for its content or policies. 


\section{Pacific Journal of Mathematics}

\section{Vol. 12 , No. 4 \\ April, 1962}

Tsuyoshi Andô, On fundamental properties of a Banach space with a cone ..... . 1163

Sterling K. Berberian, A note on hyponormal operators ................ 1171

Errett Albert Bishop, Analytic functions with values in a Frechet space . . . . . . . 1177

(Sherman) Elwood Bohn, Equicontinuity of solutions of a quasi-linear equation ............................................ 1193

Andrew Michael Bruckner and E. Ostrow, Some function classes related to the class of convex functions . . . . . . . . . . . . . . . . . . . . . . . . 1203

J. H. Curtiss, Limits and bounds for divided differences on a Jordan curve in the complex domain . . ................................. 1217

P. H. Doyle, III and John Gilbert Hocking, Dimensional invertibility . . . . . . . . 1235

David G. Feingold and Richard Steven Varga, Block diagonally dominant matrices and generalizations of the Gerschgorin circle theorem ................. 1241

Leonard Dubois Fountain and Lloyd Kenneth Jackson, A generalized solution of the boundary value problem for $y^{\prime \prime}=f\left(x, y, y^{\prime}\right) \ldots \ldots \ldots \ldots \ldots \ldots \ldots \ldots \ldots$

Robert William Gilmer, Jr., Rings in which semi-primary ideals are primary. . . . . 1273

Ruth Goodman, K-polar polynomials .......................... 1277

Israel Halperin and Maria Wonenburger, On the additivity of lattice completeness ........................................... 1289

Robert Winship Heath, Arc-wise connectedness in semi-metric spaces . . . . . . . . 1301

Isidore Heller and Alan Jerome Hoffman, On unimodular matrices . . . . . . . . . . . 1321

Robert G. Heyneman, Duality in general ergodic theory . . . . . . . . . . . . . . . 1329

Charles Ray Hobby, Abelian subgroups of p-groups . . . . . . . . . . . . . . . 1343

Kenneth Myron Hoffman and Hugo Rossi, The minimum boundary for an analytic

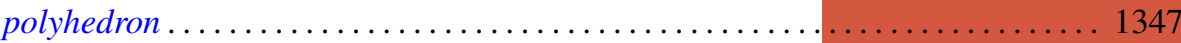

Adam Koranyi, The Bergman kernel function for tubes over convex cones ........ 1355

Pesi Rustom Masani and Jack Max Robertson, The time-domain analysis of a continuous parameter weakly stationary stochastic proces.

William Schumacher Massey, Non-existence of almost-complex structures on

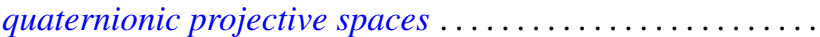

Deane Montgomery and Chung-Tao Yang, A theorem on the action of $\mathrm{SO}(3) \ldots . .1385$

Ronald John Nunke, A note on Abelian group extensions . . . . . . . . . . . . . 1401

Carl Mark Pearcy, A complete set of unitary invariants for operators generating finite $W^{*}$-algebras of type $I$

Edward C. Posner, Integral closure of rings of solutions of linear differential equations.

Duane Sather, Asymptotics. III. Stationary phase for two parameters with an application to Bessel functions.

J. Śladkowska, Bounds of analytic functions of two complex variables in domains

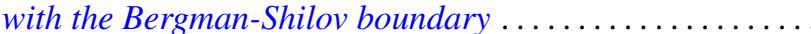

Joseph Gail Stampfli, Hyponormal operators .

George Gustave Weill, Some extremal properties of linear combinations of kernels

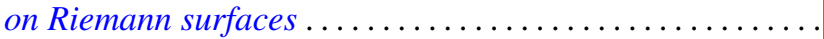

